\title{
PROCESSO DE INCLUSÃO DE CRIANÇAS COM TRANSTORNO DO ESPECTRO AUTISTA - TEA - NA EDUCAÇÃO INFANTIL E 0 PAPEL DO PSICOPEDAGOGO
}

\author{
CAROLINA PRESENZA SAMPIETRI* \\ PROFA. ME. MARIA DO CARMO GONZALEZ BORGES**
}

\begin{abstract}
RESUMO
O presente artigo tem por objetivo estudar o papel do psicopedagogo no processo de inclusão de crianças com Transtorno do Espectro Autista (TEA) na Educação Infantil. Trata-se de uma pesquisa qualitativa, realizada por meio de levantamento bibliográfico a respeito dos temas. A inclusão das crianças com TEA é garantida por leis, diretrizes e declarações, porém, ainda é distante do cotidiano das salas de aula. Neste sentido, é de grande importância compreender o papel do psicopedagogo e sua atuação e intervenções no processo de inclusão.
\end{abstract}

Palavras-chave: Inclusão. Psicopedagogia. Autismo.

PROCESS OF INCLUSION OF CHILDREN WITH AUTISTIC SPECTRUM DISORDER - TEA -
IN CHILDHOOD EDUCATION AND THE
ROLE OF PSYCHOPEDAGOGUE.

\begin{abstract}
This article aims to study the role of the Psychopedagogue in the process of including children with Autism Spectrum Disorders in Early Childhood Education. This paper is a qualitative
\end{abstract}

\footnotetext{
* Aluna do Curso de Lato Sensu em Psicopedagogia da Universidade Metodista de São Paulo.

** Professora do Curso de Lato Sensu em Psicopedagogia da Universidade Metodista de São Paulo, orientadora do Trabalho de Conclusão de Curso.
} 
research, carried out through bibliographic analysis about these themes. The inclusion of children with ASD is guaranteed by law, educational guidelines and declarations, however, it is still far from the classroom's daily life. In this sense, it is very important to understand the role of the psychopedagogue in the inclusion process.

Keywords: Inclusion. Autism. Psychopedagogy.

\section{INTRODUÇÃO}

De acordo com Costa, Pinto e Andrade (2013), a psicopedagogia nasce na Europa, no século XIX e é possível encontrar nos trabalhos de Janine Mery, psicopedagoga francesa, a história e a origem da psicopedagogia na Europa.

A partir do século XVIII, como descreve Rosa (2017), é possível identificar educadores como Pereire,Itard e Seguin que passam a se dedicar ao estudo da aprendizagem de pessoas com deficiência.

Alguns psiquiatras, ainda segundo Rosa (2017), como Montessori, influenciados pelas ideias de Itard e Seguin também se debruçam sobre as questões de aprendizagem, principalmente no que se refere à inclusão de pessoas com deficiência.

A partir de 1946 são fundados, de acordo com Mery (1985) apud Costa, Pinto e Andrade (2013), os primeiros centros psicopedagógicos, onde se reuniram os conhecimentos da psicologia, psicanálise e pedagogia voltados para o tratamento dos comportamentos das crianças, considerados socialmente inadequados.

Vê-se que a psicopedagogia, dessa forma (COSTA, PINTO e ANDRADE, 2013), surge do interesse dos profissionais da psicologia, pedagogia e psicanálise focados nos problemas de aprendizagem apresentados por crianças com deficiência.

Apesar do crescente número de discussões e estudos sobre a inclusão de crianças com deficiência, muito ainda falta quando pensamos no cotidiano das escolas e nas adaptações que as instituições escolares necessitam colocar em prática, para que a inclusão seja orgânica e efetiva. 
Com foco, aqui, nas crianças com Transtorno do Espectro Autista, o psicopedagogo aparece como profissional atuante nesse processo de inclusão, auxiliando as escolas quanto às práticas a serem adotadas para sanar a grande disparidade entre o que garantem as leis e diretrizes e o que é, de fato, oferecido.

Considera-se oportuno mencionar que o autismo foi descrito pela primeira vez, pelo médico austríaco, Leo Kanner (18941981), em 1943 (KLIN, 2006; LIRA, 2004). Segundo D’Antino, Brunoni, e Schwartzman (2015), Transtorno do Espectro Autista (TEA) é uma condição neurobiológica que ocorre com maior frequência em crianças do sexo masculino. É ocasionado por diversos fatores - multifatorial - e atinge áreas comportamentais, de interação social e de comunicação, variando o grau de severidade em cada indivíduo.

O presente trabalho tem por objetivo compreender o processo de inclusão das crianças com Transtornos do Espectro Autista e o papel do psicopedagogo, entendendo este profissional como um agente de extrema importância para garantir a efetiva inclusão da criança no espectro, bem como seu sucesso no processo de escolarização.

\section{METODOLOGIA}

O presente artigo tem como base um estudo qualitativo, realizado a partir de levantamento bibliográfico e documental, tendo em vista o caráter teórico-argumentativo do tema apresentado.

A primeira parte consistirá na concepção do Transtorno do Espectro Autista e um breve histórico do estudo do transtorno, e na descrição de leis e declarações que garantem (de maneira formal) o acesso à educação inclusiva das crianças no espectro.

Posteriormente, abordaremos o histórico da psicopedagogia no mundo e no Brasil, tratando das deficiências, do papel do psicopedagogo no ambiente escolar, com destaque para a maneira como esse profissional atua no processo de inclusão escolar efetiva de crianças com Transtorno do Espectro Autista, na Educação Infantil. 
A pesquisa recorre a Choto (2007), que destaca a soma dos conhecimentos e métodos pedagógicos e psicológicos como fator de extrema importância para a melhora do desenvolvimento da criança autista.

Assim, buscaremos, aqui, responder ao seguinte questionamento: qual deve ser o papel do psicopedagogo nas instituições escolares para que as crianças com TEA sejam efetiva e verdadeiramente inclusas, desenvolvendo plenamente suas potencialidades?

Apesar da existência de tratados e declarações - como a Declaração Universal dos Direitos Humanos (ONU, 1948) e da Declaração dos Direitos da Pessoa com Deficiência (ONU, 1975), e de leis brasileiras, como a 12.764/2012, que institui a Política Nacional de Proteção dos Direitos da Pessoa com Transtorno do Espectro Autista - que discorrem, entre outros aspectos, sobre a educação das pessoas com deficiência (sendo esta última focada, especificamente, nas pessoas com TEA), as instituições escolares ainda encontram dificuldade na inclusão dessas crianças.

Concordando com Lemos, Salomão e Agripino-Ramos (2014, p.126), “evidencia-se que as estratégias adotadas pelas professoras são, na maioria das vezes, baseadas na intuição, com pouco respaldo teórico e pouca orientação de profissionais capacitados".

Neste sentido, o psicopedagogo se apresenta como o profissional capaz de direcionar ações - tanto da escola, como das professoras e dos professores, na construção de um ambiente inclusivo - que evidenciam que é possível conviver com a heterogeneidade, tendo como foco o pleno desenvolvimento das crianças com Transtorno do Espectro Autista.

\section{TRANSTORNO DO ESPECTRO AUTISTA: UM BREVE HISTÓRICO E DEFINIÇÕES}

Como já destacado, Leo Kanner (1894-1981), médico austríaco, descreveu o autismo pela primeira vez em 1943, em seu 
artigo "Distúrbios Autísticos do Contato Afetivo", após observar em 11 crianças características como dificuldade de relacionamento, movimentos estereotipados, ecolalia, resistência a mudanças de ambiente. (KLIN, 2006; LIRA, 2004).

Kanner classificou o autismo como uma síndrome, a qual denominou de "Autismo Infantil Precoce", e descreveu, ao longo do estudo, situações em que as crianças não eram capazes de demonstrar interação ou de se relacionarem. As crianças observadas também apresentavam maior interesse em objetos do que nas pessoas ao seu redor, e, com relação aos objetos, buscavam sempre arrumá-los e organizá-los de maneira padronizada. (SILVA, 2010).

Nas décadas seguintes, anos de 1950 e 1960, muitas foram as teorias que surgiram a respeito do autismo. Em 1978, segundo Klin (2006), Michael Rutter propôs uma definição do autismo baseado nos seguintes critérios: início antes dos 03 (três) anos de idade, movimentos estereotipados, dificuldade na comunicação e desvio social. Tal definição contribuiu para que o transtorno fosse classificado, pela primeira vez, no DSM-III nos Transtornos Invasivos do Desenvolvimento (TID), na justificativa de que, no autismo, muitas áreas do funcionamento são afetadasKLIN, 2006).

De acordo com Buss, Andrade e Stoltz (2019), o TEA está incluso, atualmente, nos Transtornos do Neurodesenvolvimento, no DSM-5 (Manual Diagnóstico e Estatístico de Transtornos Mentais), após revisões nas versões anteriores do Manual, como no caso do DSM-IV-TR (publicado em 2000 e utilizado até 2013), em que o autismo estava classificado como Transtorno Global do Desenvolvimento (TGD).

Além do DSM, as versões da Classificação Estatística Internacional de Doenças e Problemas Relacionados com a Saúde também sofreram alterações no que se refere ao TEA. O CID-10, trazia sob o código F84 - Transtornos Globais do Desenvolvimento (TGD) - diversos diagnósticos, entre eles o 
Autismo Infantil (F84.0), o Autismo Atípico (F84.1), a Síndrome de Asperger (F84.5).

A última versão, atualizada pela OMS (Organização Mundial da Saúde) em junho de 2018, classifica o Transtorno do Espectro do Autismo (TEA) com o código 6A02 e, sob ele, códigos diferentes para o detalhamento do comprometimento da linguagem funcional, da deficiência intelectual associada ou não, e do grau de severidade. (ALMEIDA, 2019).

Recorremos novamente às autoras Buss, Andrade e Stoltz (2019), segundo as quais "o DSM-5 definiu três níveis de gravidade com base em dois eixos de dificuldade". Os níveis de gravidade são "Nível 3 - Exigindo apoio muito substancial", "Nível 2 - Exigindo apoio substancial", e "Nível 1 - Exigindo apoio" para os eixos "Comunicação Social" e "Comportamentos Restritivos e Repetitivos".

D’Antino, Brunoni, e Schwartzman (2015) apresentam o TEA como:

[...] condições neurobiológicas, de início precoce (antes dos 3 anos de idade), com causas multifatoriais, e que acarretam prejuízos com níveis variados de severidade, afetando as áreas da interação social, da comunicação e do comportamento. (D'ANTINO; BRUNONI; SCHWARTZMAN, 2015, p.14).

Ainda segundo Schartzman (2015), o diagnóstico do TEA é clínico, realizado a partir da análise do comportamento das crianças e de avaliações neuropsicológicas.

As pesquisadoras Buss, Andrade e Stoltz (2019) afirmam que a família é um fator importante, tanto no diagnóstico e na análise, quanto auxiliando nas intervenções. Já Almeida (2019) sinaliza que o diagnóstico pode ser realizado pela "aplicação de escalas, questionários e protocolos padronizados de observação do comportamento".

No DSM-5 ficam enfatizadas particularidades do transtorno, como comprometimento na interação social e na comu- 
nicação, hipersensibilidade ou hipossensibilidade a estímulos externos, limitado interesse em atividades, entre outras. Nem todos os casos apresentam comprometimento na linguagem oral e, portanto, não é uma característica geral (D’Antino, Brunoni, Schwartzman, 2015).

Ainda segundo o autor, pessoas com TEA podem apresentar foco restrito em determinados assuntos e, assim, os estudam a fundo, colecionando objetos. Em alguns casos, ocorre um grande apego à rotina e uma imensa dificuldade no enfrentamento de qualquer mudança; em outros, ocorrem o que D'Antino, Brunoni e Schwartzman (2015) chamam de "respostas peculiares a estímulos sensoriais", como não reagir à dor de uma queda, mas apresentam grande sensibilidade a um toque.

Estereotipias também podem ser consideradas características das crianças no espectro, a exemplo de movimentos repetitivos, e o que os autores definem como "balanço do corpo", que podem ter diferentes finalidades, como expressão e comunicação. (BOSA; BAPTISTA, 2007).

Com foco em outra especificidade, Campos e Fernandes (2016) expõem o comprometimento da linguagem das crianças no espectro, tanto no que se refere à aquisição da linguagem falada - algumas crianças não desenvolvem as habilidades verbais; outras, ainda que adquiram a linguagem falada, apresentam permanentes déficits na comunicação em razão da falta de reciprocidade social -, como no domínio da funcionalidade da língua, no que tange ao entendimento deficitário da linguagem figurada, humor, sarcasmos e linguagem corporal.

\section{GARANTIA DO ACESSO À EDUCAÇÃO}

Maia e Ferreira (2011) apud Leal (2017) ressaltam a diferença entre igualdade legal e igualdade material, sendo esta a que se concretiza no cotidiano, garantindo os mesmos direitos e oportunidades a todos, e aquela a descrita em dispositivos jurídicos.

É possível verificar, a partir da revisão bibliográfica, documentos, tratados e leis que garantem a igualdade legal, assim 
como o direito e o acesso à educação inclusiva das crianças com Transtorno do Espectro Autista, e serão descritos a seguir.

Nakayama (2019) discorre a respeito da evolução no que se refere às leis que garantem o acesso à educação às pessoas com deficiência, desde a Constituição de 1824, passando pela Constituição de 1946, que em seu texto explicita a obrigatoriedade de "um serviço da assistência educacional que assegurem aos alunos necessitados condições de eficiência escolar". (NAKAYAMA, 2019, p. 37).

A Declaração Universal dos Direitos Humanos, de 1948, ressalta o princípio de igualdade a todos os cidadãos, sem distinções. Contudo, conforme Mendes (2006), a partir da ideia de que as necessidades educacionais das crianças e jovens com deficiência seriam melhor atendidas em classes separadas, foram implantadas, no Brasil, as classes especiais.

Em 1975, conforme citado por Leal (2017), na Declaração dos Direitos da Pessoa com Deficiência, o direito de uma educação às pessoas com deficiência, que desenvolvam todas as suas capacidades e habilidades, é reforçado, embora essa educação universal não fosse uma realidade em muitos países.

Em 1994 ocorreu a Conferência Mundial de Educação Especial, em Salamanca, na Espanha, sendo que a Declaração de Salamanca, daí decorrente, definiu como princípio que os países signatários garantissem o acesso à educação às pessoas com deficiência.

Para tanto, foi adotado o Princípio da Educação Inclusiva, segundo o qual as escolas devem acomodar todas as crianças, independentemente de suas condições físicas, intelectuais, sociais, emocionais, culturais e sua integração de grupos desvantajosos ou marginalizados". (NAKAYAMA, 2019, p. 39).

Naquele mesmo ano, no Brasil, foi publicada a Política Nacional de Educação Especial, que, conforme descrito por Buss, Andrade e Stoltz (2019, p.30), incluía o TEA na descrição "condutas típicas" de seu texto. 
A Lei de Diretrizes e Bases da Educação - LDBE n ${ }^{\circ}$ 9.394/96, segundo Nakayama (2019), tendo como base a Declaração de Salamanca, destaca que a educação especial deve estar presente nas escolas regulares, podendo ser ofertados serviços de atendimento especializado em caso de necessidade.

Buss, Andrade e Stoltz (2019) mencionam, também, a Resolução CNE/CEB 2, de 2011, que institui as Diretrizes Nacionais para a Educação Especial na Educação Básica, salientando que o atendimento das necessidades educacionais especiais dos alunos deve ser realizado nas classes regulares, com professores capacitados, com as devidas adaptações por parte da instituição escolar (no que se refere ao currículo, e recursos, por exemplo), de modo que os alunos possam conviver com as heterogeneidades.

Outros documentos que tiveram o propósito de embasar a atuação tanto dos professores quanto das instituições escolares foram o documento denominado Dificuldades acentuadas de aprendizagem - Autismo, e o Documento Subsidiário à Política de Inclusão, publicados pelo Ministério da Educação (2004) e pela Secretaria de Educação Especial (2005), respectivamente (BUSS, ANDRADE e STOLT, 2019).

A Política Nacional de Educação Especial na Perspectiva da Educação Inclusiva, documento de 2008, de acordo com Nunes, Azevedo e Schmitd (2013), citados por Buss, Andrade e Stolt (2019), fez com que o acesso à escola regular, dos alunos com Transtorno do Espectro Autista, tivesse importante aumento, além de determinar o Atendimento Educacional Especializado a essas crianças, no contraturno escolar.

A Lei 12.764/2012, conforme a Cartilha dos Direitos da Pessoa com Autismo, organizada pela OAB do Distrito Federal (2015), passa a classificar as pessoas com Transtorno do Espectro Autista como pessoas com deficiência. Além disso, “[...] institui a Política Nacional de Proteção dos Direitos da Pessoa com Transtorno do Espectro Autista e garante um sistema educacional inclusivo em todos os níveis de ensino[...]". (Cartilha 
dos Direitos da Pessoa com Autismo, OAB do Distrito Federal, 2015, p.23).

A lei assegura, ainda de acordo com a Cartilha dos Direitos da Pessoa com Autismo, organizada pela OAB do Distrito Federal (2015), que os alunos com TEA possam se matricular na rede regular de ensino com atendimento especializado e auxílio de um profissional de apoio (caso seja comprovada a necessidade). A lei assevera também a capacitação dos professores, objetivando a inclusão dos alunos no espectro, inclusive no que se refere a comunicações alternativas.

A partir dos documentos mencionados acima é possível verificar que o acesso à educação é garantido às crianças com deficiência e, como foco ao presente artigo, às crianças com Transtorno do Espectro Autista, por meio de instrumentos legais. Porém, quando adentramos o cotidiano das escolas, vemos que ainda há uma grande dificuldade quanto à efetiva inclusão dessas crianças.

Nakayama (2019), discorrendo a respeito da Educação Inclusiva, afirma que:

A presença das crianças nas escolas é, sem dúvida, o primeiro passo da sua inclusão, mas elas necessitam oportunidade de participação em todas as atividades que lhe proporcione acesso ao aprendizado, caso contrário continuarão desligadas do processo educacional. (NAKAYAMA, 2019, p. 57).

Trazendo a afirmação de Nakayama (2019) acerca da inclusão das crianças com TEA na Educação Infantil, não basta que as crianças estejam inseridas no contexto escolar, mas que sejam capazes de desenvolver suas potencialidades, habilidades e adquirir reais conhecimentos.

Nesse processo, o psicopedagogo se apresenta como o profissional capaz de direcionar as ações, tanto da escola como das professoras e dos professores, na construção de um ambiente efetivamente inclusivo. 


\section{PSICOPEDAGOGIA E INCLUSÃO}

Costa, Pinto e Andrade (2013), Bossa (2019) e Rosa (2017) apontam os estudos realizados por Janine Mery a respeito da psicopedagogia e identificam sua origem a partir do século XIX, período em que alguns estudiosos passaram a focar nos problemas de aprendizagem das crianças com deficiência. Entre os pesquisadores destacam-se Pereire, Itard e Seguin, além de psiquiatras, como Maria Montessori - que se debruçam sobre as questões de aprendizagem, principalmente no que se refere à inclusão de pessoas com deficiência (ROSA, 2017).

A partir de 1946 são fundados, segundo Mery (1985) apud Costa, Pinto e Andrade (2013), os primeiros centros psicopedagógicos, onde se reuniram os conhecimentos da psicologia, psicanálise e pedagogia, para o tratamento dos comportamentos das crianças, considerados socialmente inadequados.

A psicopedagogia, como descrito por Costa, Pinto e Andrade (2013), surge do interesse dos profissionais da psicologia, pedagogia e psicanálise sobre os problemas de aprendizagem apresentados por crianças com deficiência.

No Brasil, a partir da década de 1980, de acordo com Bossa (2019), a patologização dos problemas de aprendizagem dá lugar a uma perspectiva sociopolítica, entendendo o fracasso escolar como um conjunto de fatores que não somente os orgânicos. Neste sentido, ocupando-se da aprendizagem humana, a psicopedagogia passa a preocupar-se com os problemas de aprendizagem, sob uma ótica que vai além da psicologia e da pedagogia.

No contexto escolar, a psicopedagogia tem o papel de "[...] analisar um processo que inclui questões metodológicas, relacionais e socioculturais, englobando o ponto de vista de quem ensina e de quem aprende, abrangendo a participação da família e da sociedade" (BOSSA, 2019, p.117).

Lemos, Salomão e Agripino-Ramos (2014) e Buss, Andrade e Stoltz (2019) realizaram pesquisas a respeito da inclusão de crianças com Transtorno do Espectro Autista no ambiente es- 
colar. Essas, realizaram entrevistas com professoras do Centro Municipal de Educação Infantil Jardim, na cidade de Curitiba, no Paraná; aquelas, observando o cotidiano de professoras de duas escolas particulares da cidade de João Pessoa, na Paraíba.

Embora em contextos diferentes, é possível perceber que, em ambas as pesquisas, os resultados apontam a dificuldade da real inclusão das crianças no ambiente escolar, principalmente no que se refere à ação das professoras em sala de aula, que possuem pouco conhecimento para o atendimento das crianças com TEA - recursos, métodos, interação do autista com o restante da sala, entre outros fatores.

Neste sentido, algumas ações que podem ser implementadas na instituição escolar pelo profissional da psicopedagogia são: a análise e a reformulação de currículos, conteúdos e sistemas avaliativos, a orientação de professores, coordenação e direção a respeito das formas de aprendizagem, o diálogo entre escola e família. (BOSSA, 2019).

Ainda segundo Bossa (2019), o trabalho do psicopedagogo se dá em caráter clínico e preventivo - clínico porque se preocupa com o processo de aprendizagem e o profissional busca compreender as falhas que podem ocorrer. $\mathrm{O}$ caráter preventivo pode ser percebido em três níveis de trabalho: o primeiro, na orientação de professores a respeito das questões didático-metodológicas, além de orientações aos pais; o segundo, elaborando planos de intervenção baseados no diagnóstico institucional, avaliando, junto aos professores, o currículo a fim de diminuir os problemas de aprendizagem; o terceiro nível, com procedimentos clínicos, visa eliminar os problemas de aprendizagem.

Com foco no atendimento às crianças com TEA, Sousa et al. (2018) reforçam que o papel do psicopedagogo é de extrema importância no sentido de que tendo em vista as possibilidades de aprendizagem e as capacidades das crianças, atua em conjunto com a equipe multidisciplinar e com os pais/responsáveis e professores, selecionando os estímulos que farão parte do 
processo de aprendizagem e serão melhor recebidos. É, também, papel desse profissional, analisar a criança no que tange às suas preferências, interesses, e, assim, buscar novas formas de aprendizagem.

Para Nascimento e Souza (2018):

[...] o papel do Psicopedagogo apresenta-se com grande relevância, visto que, enquanto profissional voltado aos aspectos da aprendizagem, possui habilidades que favorecem a aplicação de estratégias de ensino através de um olhar mais apurado”. (SOUZA, NASCIMENTO, 2018, p. 165).

Sousa et al. (2018) destacam dois métodos de intervenções psicopedagógicas para o melhor atendimento das crianças no espectro, sendo eles o ABA e o TEACCH.

$\mathrm{O}$ TEACCH (Treatment and Education of Autistic and Related Communication Handcapped) tem por objetivo desenvolver autonomia, independência e a comunicação das crianças que possuem o transtorno, a fim de que possam se relacionar.

O TEACCH "é um instrumento de apoio para ensinar o que vem antes, o que acontece depois, possibilitando o planejamento de ações e seu encadeamento em uma sequência de trabalhos". (SOUZA et al., 2018, p. 256).

O ABA (Aplied Behavior Analysis), outro método de intervenção destacado por Sousa et al. (2018), objetiva analisar e avaliar as possíveis relações entre o ambiente, o comportamento humano e a aprendizagem. Nessa linha de raciocínio,

Nascimento e Souza (2018) afirmam que a aplicação do método de avaliação do comportamento depende de uma cuidadosa observação das crianças com TEA e o planejamento de intervenções que tenham por objetivo o desenvolvimento de habilidades de comunicação, motoras, sociais, cotidianas e de aprendizagem.

Azevedo, Nunes e Schmidt (2013) apontam medidas consideradas importantes no processo de escolarização das crianças, como o uso de tecnologias assistivas e elaboração de planos de ensino individualizados como auxiliares no processo de apren- 
dizagem das crianças com TEA, que servem como base para o planejamento pedagógico e a atuação dos professores.

\section{CONSIDERAÇÕES FINAIS}

É crescente o número de discussões e estudos sobre a inclusão de crianças com deficiência no Brasil e no mundo. Ainda assim, quando a questão da inclusão se aproxima do cotidiano das escolas, há uma grande disparidade entre teoria e prática.

A existência de leis e declarações que discorrem sobre a inclusão das crianças com deficiência - como, por exemplo, a lei 12.764/2012, que institui a Política Nacional de Proteção dos Direitos da Pessoa com Transtorno do Espectro Autista -, não garante a real inclusão das crianças, tampouco o desenvolvimento de suas potencialidades e capacidades.

Isso ocorre porque as escolas, em grande parte, pouco conhecem sobre o Transtorno do Espectro Autista e não dispõem de profissionais capacitados, que atuem de maneira efetiva no processo de inclusão.

Neste sentido, procuramos, no presente artigo, entender o papel do psicopedagogo nas instituições escolares, para que as crianças com TEA sejam efetiva e verdadeiramente inclusas, desenvolvendo plenamente suas potencialidades.

O psicopedagogo se apresenta como o profissional capaz de direcionar ações, tanto da escola, como das professoras e dos professores, seja a partir da reformulação de currículos, conteúdos e sistemas avaliativos, da elaboração de planos de ensino individualizados, ou da orientação de professores, coordenação e direção a respeito das formas de aprendizagem e da seleção de recursos e estímulos que melhor se adequem ao processo de aprendizagem da criança com TEA, além do diálogo entre escola e família.

Além disso, é possível que o psicopedagogo faça uso de intervenções psicopedagógicas para o melhor atendimento das crianças que possuem esse espectro, como o TEACCH e o ABA, 
a fim de lhes proporcionar independência, autonomia e desenvolvimento de habilidades de comunicação, motoras e sociais.

\section{REFERÊNCIAS}

AZEVEDO, Mariana Queiróz Orrico; NUNES, Débora Regina de Paula; SCHMIDT, Carlo. Inclusão educacional de pessoas com autismo no Brasil: uma revisão da literatura. 2013. Disponível em: <https://periodicos. ufsm.br/educacaoespecial/article/view/10178>. Acesso em: 15 maio 2020.

BAPTISTA, C.R. BOSA, C. (Org). Autismo e Educação: Reflexões e Propostas de Intervenção. Dados eletrônicos. Porto Alegre, Artmed, 2007. Disponível em: <https://books.google.com.br/books?hl=pt-BR\&lr=\&id=ffG_UEHQ_GMC\&oi=fnd\&pg=PA6\&dq=AUTISMO

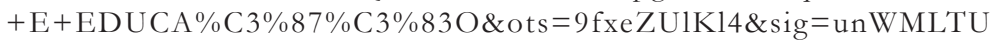
S0dQ7kJ7gWXSB_HoCRWI\#v= onepage\&q=AUTISMO $\% 20 \mathrm{E} \% 20$ EDUCA \%C3\%87\%C3\%83O\&f=false>. Acesso em: 09 maio 2020.

BOSSA, Nádia A. A Psicopedagogia no Brasil. Contribuições a Partir da Prática. Rio de Janeiro, Editora Wak, 2019.

CAMPOS, L. K.; FERNANDES, F.D.M. Perfil escolar e as habilidades cognitivas e de linguagem de crianças e adolescentes do espectro do autismo. CoDAS. São Paulo, v.28, n.3, p.234-243, 2016. Disponível em: <https:// www.scielo.br/pdf/codas/v28n3/2317-1782-codas-2317-178220162015023. pdf $>$ Acesso em: 05 jul. 2020.

COSTA, Ana A.; PINTO, Telma Maranhão G.; ANDRADE, Márcia Siqueira de. Análise Histórica do Surgimento da Psicopedagogia no Brasil. Online Revista Multidisciplinar e de Psicologia. V.7. N.20. 2013. Disponível em <https://idonline.emnuvens.com.br/id/article/view/234/258>. Acesso em: 31 mar. 2020.

CHOTO, M. C. Autismo infantil: el estado de la cuestión. Revista Ciencias Sociales Universidad de Costa Rica, v.116, n.2, p.169-180, 2007. Disponível em: <https://pdfs.semanticscholar.org/4a5f/7bf58e0a2c839f9542180dae8782 0ad33b83.pdf>. Acesso em: 05 jul. 2020.

D'ANTINO, Maria Eloisa Famá; BRUNONI, Décio; SCHWARTZMAN, José Salomão. Contribuições para a inclusão escolar de alunos com necessidades especiais: Estudos interdisciplinares em educação e saúde em alunos com Transtorno do Espectro do Autismo no município de Barueri, SP. Programa de Pós-graduação em Distúrbios do Desenvolvimento da Universidade Presbite- 
riana Mackenzie, 2015. Disponível em: <https://www.mackenzie.br/fileadmin/ ARQUIVOS/Public/6-pos-graduacao/upm-higienopolis/mestrado-doutorado/ disturbios_desenvolvimento/2018/periodicos/Contribui $\%$ C3\%A7\%C3\%B5es_ para_a_inclus $\%$ C3\%A3o_escolar_de_alunos_com_necessidades_especiais.pdf $>$. Acesso em: 31 mar. de 2020.

DECLARAÇÃO UNIVERSAL DOS DIREITOS HUMANOS. Assembleia Geral das Nações Unidas em Paris. 10 dez. 1948. Disponível em: http://www. mp.go.gov.br/portalweb/hp/7/docs/declaracao_universal_dos_direitos_do_homem.pdf. Acesso em: 15 maio 2020.

DECLARAÇÃO DOS DIREITOS DO DEFICIENTE MENTAL, 1975. Disponível em: http://www.dhnet.org.br/direitos/sip/onu/deficiente/lex61. htm. Acesso em 09 de maio de 2020.

KLIN, A. Autismo e síndrome de Asperger: uma visão geral. Revista Brasileira de Psiquiatria. São Paulo, v.28, 2006. Disponível em: https://www. scielo.br/pdf/rbp/v28s1/a02v28s1.pdf. Acesso em: 09 maio 2020.

LIRA, Solange M. Escolarização de Alunos Autistas: Histórias de Sala de Aula. Dissertação de Mestrado em Educação. Centro de Educação e Humanidades. Universidade do Estado do Rio de Janeiro. Rio de Janeiro, 2004. Disponível em: http://www.proped.pro.br/teses/teses_pdf/Solange_Maria_de_Lira-ME.pdf. Acesso em: 03 maio 2020.

LEAL, Daniela. Educação inclusiva pós - Jomtien e Salamanca: rumos, avanços e desafios. In: LEAL, Daniela (Org.). História, memória e práticas da inclusão escolar. Curitiba: InterSaberes, 2017.

LEMOS, Emellyne L. de M. Dias; SALOMÃO, Nádia Maria Ribeiro; AGRIPINO-RAMOS, Cibele Shirley. Inclusão de Crianças Autistas: Um Estudo sobre Interações Sociais no Contexto Escolar. Revista Brasileira de Educação Especial, Marília, v.20, n.1, p-117-130, 2014. Disponível em: <http://www. scielo.br/scielo.php?script $=$ sci_arttext\&pid $=$ S1413-65382014000100009\&lng $=$ pt\&nrm=iso $>$. Acesso em: 09 maio 2020 .

MENDES, Enicéia Gonçalves. A radicalização do debate sobre inclusão escolar no Brasil. Rev. Bras. Educ., Rio de Janeiro, vol.11 no.33, 2006. Disponível em: <https://www.scielo.br/pdf/rbedu/v11n33/a02v1133.pdf>.

MINISTÉRIO DA SAÚDE. Diretrizes de Atenção à Reabilitação da Pessoa com Transtornos do Espectro do Autismo (TEA). Disponível em: <https://bvsms.saude.gov.br/bvs/publicacoes/diretrizes_atencao_reabilitacao_pessoa_autismo.pdf>. Brasília, 2014. Acesso em: 31 mar. de 2020. 
NAKAYAMA, Antônia Maria. Educação Inclusiva: Fundamentos e Perspectivas. Curitiba: Appris, 2019.

ORDEM DOS ADVOGADOS DO BRASIL - SECCIONAL DISTRITO FEDERAL. Comissão de Defesa dos Direitos da Pessoa com Deficiência. Cartilha de Direitos da Pessoa com Autismo, 2015.

ROSA, Kaciana Nascimento da Silva. Os primórdios das ideias de inclusão: Pereira, Itard, Séguin e Montessori. In: LEAL, Daniela (Org.). História, memória e práticas da inclusão escolar. Curitiba: InterSaberes, 2017.

SILVA, Emanuelle C. C. da. Autismo e Troca Social: Contribuições de uma Abordagem Microgenética. Tese de Doutorado em Psicologia Cognitiva. Centro de Filosofia e Ciências Humanas. Departamento de Psicologia. Universidade Federal de Pernambuco. Recife, 2010. Disponível em: <https://repositorio. ufpe.br/bitstream/123456789/8010/1/arquivo1355_1.pdf $>$. Acesso em: 03 maio 2020 .

SOUSA, Luciano Dias de. et. al. A intervenção Psicopedagógica no Processo Ensino Aprendizagem do Autista. Revista Transformar, v.12, n.1. Itaperuna, 2018. Disponível em: <http://www.fsj.edu.br/transformar/index.php/transformar/article/view/135/118>. Acesso em: 15 maio 2020. 